TRANSACTIONS OF THE

AMERICAN MATHEMATICAL SOCIETY

Volume 361, Number 1, January 2009, Pages 547-560

S 0002-9947(08)04536-4

Article electronically published on July 24, 2008

\title{
SOBOLEV ORTHOGONAL POLYNOMIALS: BALANCE AND ASYMPTOTICS
}

\author{
MANUEL ALFARO, JUAN JOSÉ MORENO-BALCÁZAR, ANA PEÑA \\ AND M. LUISA REZOLA
}

Abstract. Let $\mu_{0}$ and $\mu_{1}$ be measures supported on an unbounded interval and $S_{n, \lambda_{n}}$ the extremal varying Sobolev polynomial which minimizes

$$
\langle P, P\rangle_{\lambda_{n}}=\int P^{2} d \mu_{0}+\lambda_{n} \int P^{\prime 2} d \mu_{1}, \quad \lambda_{n}>0,
$$

in the class of all monic polynomials of degree $n$. The goal of this paper is twofold. On the one hand, we discuss how to balance both terms of this inner product, that is, how to choose a sequence $\left(\lambda_{n}\right)$ such that both measures $\mu_{0}$ and $\mu_{1}$ play a role in the asymptotics of $\left(S_{n, \lambda_{n}}\right)$. On the other hand, we apply such ideas to the case when both $\mu_{0}$ and $\mu_{1}$ are Freud weights. Asymptotics for the corresponding $S_{n, \lambda_{n}}$ are computed, illustrating the accuracy of the choice of $\lambda_{n}$.

\section{INTRODUCTION}

One of the central problems in the analytic theory of orthogonal polynomials is the study of their asymptotic behavior. In this paper we are concerned with the asymptotic properties of Sobolev orthogonal polynomials, that is, polynomials orthogonal with respect to an inner product involving derivatives. In this sense, given $\mu_{0}$ and $\mu_{1}$ finite Borel measures supported on an interval $I \subset \mathbb{R}$ and $\lambda>0$, we consider the Sobolev inner product

$$
\langle P, Q\rangle_{\lambda}=\int P Q d \mu_{0}+\lambda \int P^{\prime} Q^{\prime} d \mu_{1}
$$

in the space of all polynomials with real coefficients.

We denote by $P_{n, \mu_{0}}, P_{n, \mu_{1}}$ and $S_{n, \lambda}$ the corresponding monic polynomials orthogonal with respect to $\mu_{0}, \mu_{1}$ and $\langle\cdot, \cdot\rangle_{\lambda}$, respectively.

Received by the editors June 26, 2006 and, in revised form, October 19, 2006 and April 26, 2007.

2000 Mathematics Subject Classification. Primary 42C05.

Key words and phrases. Asymptotics, varying Sobolev inner products, potential theory, Mhaskar-Rakhmanov-Saff numbers, Freud weights.

The first author was partially supported by MEC of Spain under Grant MTM2006-13000-C0303, FEDER funds (EU), and the DGA project E-64 (Spain).

The second author was partially supported by MEC of Spain under Grant MTM2005-08648C02-01 and Junta de Andalucía (FQM229 and excellence projects FQM481, PO6-FQM-1735).

The third author was partially supported by MEC of Spain under Grants MTM 2004-03036 and MTM2006-13000-C03-03, FEDER funds (EU), and the DGA project E-64, Spain.

The fourth author was partially supported by MEC of Spain under Grant MTM2006-13000C03-03, FEDER funds (EU), and the DGA project E-64 (Spain). 
Let $\mu_{0}$ and $\mu_{1}$ be measures compactly supported on $\mathbb{R}$. Whether $\left(\mu_{0}, \mu_{1}\right)$ is a coherent pair, which means that there exist nonzero constants $\sigma_{n}$ such that the corresponding monic polynomials satisfy for each $n$,

$$
P_{n, \mu_{1}}=\frac{P_{n+1, \mu_{0}}^{\prime}}{n+1}+\sigma_{n} \frac{P_{n, \mu_{0}}^{\prime}}{n}
$$

or, if $\mu_{0}$ and $\mu_{1}$ fulfill much milder conditions, i.e., they belong to the well-known Szegö class, then it has been established (see [9] and [8] that the ratio asymptotics

$$
\lim _{n \rightarrow \infty} \frac{S_{n, \lambda}(z)}{P_{n, \mu_{1}}(z)}=\frac{2}{\varphi^{\prime}(z)}
$$

holds uniformly on compact subsets of $\overline{\mathbb{C}} \backslash[-1,1]$, where $\varphi(z)=z+\sqrt{z^{2}-1}$ with $\sqrt{z^{2}-1}>0$ when $z>1$. In other words, the measure $\mu_{0}$ does not appear explicitly within the asymptotic expression.

Nevertheless, a closer look at the inner product (11) explains the "dominance" of the measure $\mu_{1}$ in the asymptotics: the derivative makes the leading coefficient of the polynomials in the second integral of (1) be multiplied by the degree of the polynomial. Thus, if we want both measures to have an impact on the behavior of the polynomials for $n \rightarrow \infty$, it seems natural to "balance" the inner product, that is, to compensate both integrals by introducing a varying parameter $\lambda_{n}$.

In a general framework, we consider the varying Sobolev inner product $\langle P, Q\rangle_{\lambda_{n}}$. We denote by $S_{n, \lambda_{n}}$ the monic polynomial which minimizes the expression $\left\langle Q_{n}, Q_{n}\right\rangle_{\lambda_{n}}$ in the class of all monic polynomials $Q_{n}$ of degree $n$.

Concerning the choice of the varying parameter $\lambda_{n}$, it is interesting to write the expression of the Sobolev inner product in terms of monic polynomials, that is,

$$
\left\langle Q_{n}, Q_{n}\right\rangle_{\lambda_{n}}=\int\left(Q_{n}\right)^{2} d \mu_{0}+\lambda_{n} n^{2} \int\left(\frac{Q_{n}^{\prime}}{n}\right)^{2} d \mu_{1}
$$

In this expression each integral on the right hand side is bounded from below by $\int P_{n, \mu_{0}}^{2} d \mu_{0}$ and $\int P_{n-1, \mu_{1}}^{2} d \mu_{1}$, respectively, as long as $Q_{n}$ is a monic polynomial of degree $n$.

If the measures $\mu_{0}$ and $\mu_{1}$ are supported on the same bounded interval where they satisfy the Szegö condition, then $\int P_{n, \mu_{0}}^{2} d \mu_{0}$ behaves as $\int P_{n-1, \mu_{1}}^{2} d \mu_{1}$, when $n \rightarrow \infty$. More precisely, the ratio $\frac{\int P_{n, \mu_{0}}^{2} d \mu_{0}}{\int P_{n-1, \mu_{1}}^{2} d \mu_{1}}$ has a limit. Therefore, in order to balance both terms in (2) it is natural to keep $\lambda_{n} n^{2}$ bounded.

In fact, it was proved in [1] that if $\left(\lambda_{n}\right)$ is a decreasing sequence of positive real numbers such that $\lim _{n} \lambda_{n} n^{2} \in(0,+\infty)$, then

$$
\lim _{n \rightarrow \infty} \frac{S_{n, \lambda_{n}}(z)}{R_{n}(z)}=1
$$

locally uniformly in $\overline{\mathbb{C}} \backslash[-1,1]$, where $\left(R_{n}\right)$ is the sequence of monic polynomials orthogonal with respect to a measure constructed as a certain combination of the measures $\mu_{0}$ and $\mu_{1}$.

Let us consider now that the measures $\mu_{0}$ and $\mu_{1}$ are supported on an unbounded interval. There are many asymptotic results (strong asymptotics) for the monic polynomials $S_{n, \lambda}$ orthogonal with respect to the inner product (11) for a fixed $\lambda$; see for instance [2] and [1] for coherent pairs, 3] and 4] for Freud weights and, more recently, the survey [7]. But as far as we know, nothing has been said about 
asymptotics in the balanced case. In this sense, the first question that should be answered is: what is the appropriate choice for the sequence $\left(\lambda_{n}\right)$ ? We understand by this a sequence of parameters for which the polynomials $S_{n, \lambda_{n}}$ exhibit a nontrivial asymptotic behavior, depending on both measures $\mu_{0}$ and $\mu_{1}$. One of the goals of this paper is to raise the point that $\lambda_{n}=n^{-2}$ is not, in general, the right choice when the support of $\mu_{0}$ and $\mu_{1}$ is unbounded.

The structure of the paper is as follows. In Section 2, we use heuristic arguments, based on potential theory, pertaining to the "size" of $\lambda_{n}$ in order to achieve an appropriate "balancing". In this sense, the Mhaskar-Rakhmanov-Saff numbers turn out to be a powerful tool. On account of the above results, in Section 3 we obtain asymptotics for Sobolev polynomials and their norms for a particular case of Freud weights, which illustrates that the choice of $\lambda_{n}$ is accurate.

\section{Selection of the parameters}

We point out some heuristic reasoning concerning the asymptotic behavior of the parameters $\lambda_{n}$ in order to balance both terms in the varying Sobolev inner product $\langle P, Q\rangle_{\lambda_{n}}$.

Firstly, we recall some basic tools from classical potential theory with an external field which will be used later on.

Let $\mu$ be a probability measure with support in a closed set $\Sigma$ of the complex plane. Recall that, the logarithmic potential $V^{\mu}$ associated with $\mu$ is defined by $V^{\mu}(z)=-\int \log |z-t| d \mu(t)$. Let us assume that $w(z)=e^{-Q(z)}$ is an admissible and continuous weight function in $\Sigma$. It is well known that there exists a unique probability measure $\mu_{w}$, called the extremal or equilibrium measure associated with $w$, minimizing the weighted energy:

$$
I_{w}(\mu)=\int_{\Sigma}\left(V^{\mu}(z)+2 Q(z)\right) d \mu(z)
$$

for all probability measures with support in $\Sigma$. This measure $\mu_{w}$ is compactly supported and there exists a constant $F_{w}$ (the modified Robin constant of $\Sigma$ ) such that $V^{\mu_{w}}(z)+Q(z)=F_{w}$ quasi-everywhere on $\operatorname{supp}\left(\mu_{w}\right)$; see [14, Theorem 1.3, p. 27]. Moreover, if $Q$ is an even function with some additional properties, then it can be deduced that

$$
\left\|w^{n} Q_{n}\right\|_{L_{\infty}(\Sigma)}=\left\|w^{n} Q_{n}\right\|_{L_{\infty}\left(\operatorname{supp}\left(\mu_{w}\right)\right)}
$$

for every polynomial $Q_{n}$ of degree $\leq n$; see [14, p. 203]. As a straightforward application of these results, we can obtain for weighted polynomials a symmetric compact interval on which its supremum norm lives; more precisely, we have

$$
\left\|w Q_{n}\right\|_{L_{\infty}(\Sigma)}=\left\|w Q_{n}\right\|_{L_{\infty}\left(\left[-a_{n}, a_{n}\right]\right)}
$$

for every polynomial $Q_{n}$ of degree $\leq n$. The number $a_{n}(n \geq 1)$ is the so-called $n$th Mhaskar-Rakhmanov-Saff number for $Q$, that is, the positive root of the equation

$$
n=\frac{2}{\pi} \int_{0}^{1} \frac{a_{n} t Q^{\prime}\left(a_{n} t\right)}{\sqrt{1-t^{2}}} d t .
$$

The link between the equilibrium measure and the asymptotics of orthogonal polynomials is given by the following observation: for a polynomial $Q_{n}(z)=$ $\left(z-c_{1}\right)\left(z-c_{2}\right) \ldots\left(z-c_{n}\right)$ we can write $\log \left|Q_{n}(z)\right|=-n V^{\nu_{n}}(z)$, where $\nu_{n}$ is the normalized counting measure on the zeros of $Q_{n}$, that is, $\nu_{n}=\frac{1}{n} \sum_{i=1}^{n} \delta_{c_{i}}$. Then $\left|w^{n}(z) Q_{n}(z)\right|^{1 / n}=e^{-\left(V^{\nu_{n}}(z)+Q(z)\right)}$. 
If we denote by $T_{n, w}$ the $n$th weighted monic Chebyshev polynomial corresponding to $w$, that is, the solution of the extremal problem

$$
\inf \left\{\left\|w^{n} Q_{n}\right\|_{L_{\infty}(\Sigma)} ; Q_{n}(z)=z^{n}+\ldots\right\}
$$

then

$$
\lim _{n \rightarrow \infty}\left\|w^{n} T_{n, w}\right\|_{L_{\infty}(\Sigma)}^{1 / n}=e^{-F_{w}}
$$

see [14, Theorem 3.1, p. 163].

Keeping in mind our balance problem, we are interested in the asymptotic behavior of the $L_{2}$-norm in $[-1,1]$ with varying weights. Since

$$
\lim _{n \rightarrow \infty}\left(\frac{\left\|w^{n} Q_{n}\right\|_{L_{\infty}([-1,1])}}{\left\|w^{n} Q_{n}\right\|_{L_{2}([-1,1])}}\right)^{1 / n}=1
$$

for every polynomial of degree $n$ (see [15, Theorem 3.2.1, p. 65]), the asymptotic extremality of $\left\|w^{n} Q_{n}\right\|_{L_{2}([-1,1])}^{1 / n}$ can be thought as the corresponding one of $\left\|w^{n} Q_{n}\right\|_{L_{\infty}([-1,1])}^{1 / n}$. In fact, if we denote by $P_{n, w^{n}}$ the solution of the extremal problem

$$
\inf \left\{\left\|w^{n} Q_{n}\right\|_{L_{2}([-1,1])} ; Q_{n}(z)=z^{n}+\ldots\right\},
$$

then it can be deduced (see [15, Theorem 3.3.3, p. 78]) that there exists

$$
\lim _{n \rightarrow \infty}\left\|w^{n} P_{n, w^{n}}\right\|_{L_{2}([-1,1])}^{1 / n} .
$$

From now on, $f_{n}(x) \sim g_{n}(x)$ in a domain $D$ will denote that there are positive constants $C_{1}, C_{2}$ such that $C_{1} g_{n}(x) \leq f_{n}(x) \leq C_{2} g_{n}(x)$, for all $x \in D$ and $n$ large enough.

In relation with our problem, we consider the varying Sobolev inner product $\langle\cdot, \cdot\rangle_{\lambda_{n}}$ where $d \mu_{i}=W^{2}(x) d x, i=0,1$. Here, we assume that $W(x)=e^{-Q(x)}$ is a weight function where $Q: I=(-c, c) \rightarrow[0,+\infty)$ is a convex, smooth, and even function with $Q\left(c^{-}\right)=+\infty=Q\left((-c)^{+}\right)$and $Q(x)=0$ only for $x=0$ (we take $Q$ an even function for simplicity). For these weights $W$, see [5, Theorem 4.1, p. 95], the $L_{2}$-norm on $I$ for weighted polynomials is asymptotically equivalent to the $L_{2}$-norm on a compact interval. More precisely,

$$
\left\|W Q_{n}\right\|_{L_{2}\left(\left[-a_{n+1}, a_{n+1}\right]\right)} \leq\left\|W Q_{n}\right\|_{L_{2}(I)} \leq \sqrt{2}\left\|W Q_{n}\right\|_{L_{2}\left(\left[-a_{n+1}, a_{n+1}\right]\right)}
$$

holds for every $n$ and every polynomial $Q_{n}$ with degree $\leq n$, where $a_{n}$ are the Mhaskar-Rakhmanov-Saff numbers associated with $Q$.

From (44), we deduce that for every polynomial $Q_{n}(x)=x^{n}+\ldots$,

$$
\begin{aligned}
& \left\langle Q_{n}, Q_{n}\right\rangle_{\lambda_{n}} \sim \int_{-a_{n+1}}^{a_{n+1}} Q_{n}^{2}(x) W^{2}(x) d x+\lambda_{n} \int_{-a_{n+1}}^{a_{n+1}}\left(Q_{n}^{\prime}(x)\right)^{2} W^{2}(x) d x \\
& =a_{n+1}\left[\int_{-1}^{1} Q_{n}^{2}\left(a_{n+1} t\right) W^{2}\left(a_{n+1} t\right) d t+\lambda_{n} \int_{-1}^{1}\left(Q_{n}^{\prime}\left(a_{n+1} t\right)\right)^{2} W^{2}\left(a_{n+1} t\right) d t\right] \\
& =a_{n+1}^{2 n+1}\left[\int_{-1}^{1} U_{n}^{2}(t) W^{2}\left(a_{n+1} t\right) d t+\frac{\lambda_{n} n^{2}}{a_{n+1}^{2}} \int_{-1}^{1} V_{n-1}^{2}(t) W^{2}\left(a_{n+1} t\right) d t\right],
\end{aligned}
$$

where $U_{n}$ and $V_{n-1}$ are monic polynomials of degree $n$ and $n-1$, respectively.

Observe that (5) remains true if we take $d \mu_{i}=L_{i} W^{2}(x) d x, i=0,1$, where $L_{0}$ and $L_{1}$ are any positive constants. At first sight, the presence of the constants $L_{i}$ could seem irrelevant, but in the next section it will allow us to give an alternative reading to explain why our selection of $\lambda_{n}$ is accurate. 
Therefore, in order to balance both terms in (5), it is reasonable to require the following:

i) $\lambda_{n} n^{2} \sim a_{n+1}^{2}$;

ii) the asymptotic extremality of the $L_{2}\left(W^{2}\left(a_{n+1} t\right),[-1,1]\right)$-norm for monic polynomials of degree $n$ behaves as the corresponding one of degree $n-1$.

The previous results about potential theory lead us to think that a sufficient condition to get ii) is

$$
W^{1 / n}\left(a_{n+1} t\right) \sim w(t), \quad \forall t \in(-1,1),
$$

where $w$ is an admissible and continuous weight function.

Concerning the choice of the parameters $\lambda_{n}$ observe that, when the support of the measures $\mu_{0}$ and $\mu_{1}$ is unbounded, the size of $\lambda_{n}$ as $n^{-2}$ is not the right one, in general. If the weight satisfies (6), the choice of the parameters depends on the distribution of the measure $W^{2}(t) d t$, that is, on the corresponding MhaskarRakhmanov-Saff numbers.

We would like to point out that these ideas can also be applied in a more general framework. Indeed, consider a Sobolev inner product with two different weights, $W_{0}^{2}$ and $W_{1}^{2}$, which are linked in such a way so that $\langle\cdot, \cdot\rangle_{\lambda_{n}}$ can be expressed in terms of only one weight (either $W_{0}^{2}$ or $W_{1}^{2}$ ) satisfying condition (6)). Actually, important examples in this situation are the Hermite coherent pairs. Notice that if the pair of measures $\left(W_{0}^{2}, W_{1}^{2}\right)$ constitutes a Hermite symmetrically coherent pair (see [2] and [11]), then either

$$
\begin{aligned}
& \text { I: } W_{0}^{2}(x)=\left(x^{2}+a^{2}\right) e^{-x^{2}} \quad \text { and } \quad W_{1}^{2}(x)=e^{-x^{2}}, a \in \mathbb{R}, \quad \text { or } \\
& \text { II: } \quad W_{0}^{2}(x)=e^{-x^{2}} \text { and } W_{1}^{2}(x)=\frac{e^{-x^{2}}}{x^{2}+a^{2}}, \quad a \in \mathbb{R} \backslash\{0\} .
\end{aligned}
$$

In both cases we have

$$
\left\langle Q_{n}, Q_{n}\right\rangle_{\lambda_{n}}=\int_{\mathbb{R}}\left[Q_{n}^{2}(x)\left(x^{2}+a^{2}\right)\right] W_{1}^{2}(x) d x+\lambda_{n} \int_{\mathbb{R}}\left(Q_{n}^{\prime}(x)\right)^{2} W_{1}^{2}(x) d x,
$$

and it is not difficult to check that

$$
\frac{\left\langle Q_{n}, Q_{n}\right\rangle_{\lambda_{n}}}{a_{n+2}^{2 n+3}} \sim \int_{-1}^{1} U_{n+1}^{2}(t) W_{1}^{2}\left(a_{n+2} t\right) d t+\frac{\lambda_{n} n^{2}}{a_{n+2}^{4}} \int_{-1}^{1} V_{n-1}^{2}(t) W_{1}^{2}\left(a_{n+2} t\right) d t,
$$

where in each case, the $a_{n}$ are the Mhaskar-Rakhmanov-Saff numbers for the corresponding weight $W_{1}$, and $U_{n+1}$ and $V_{n-1}$ are monic polynomials of degree $n+1$ and $n-1$, respectively.

Since $\frac{a_{n}}{\sqrt{n}} \rightarrow \sqrt{2}$, observe that

$$
\lim _{n \rightarrow \infty} W_{1}^{1 / n}\left(a_{n+2} t\right)=e^{-t^{2}}, \quad \forall t \in(-1,1)
$$

and therefore, according to the theory stated above, the adequate choice of $\lambda_{n}$ should be $\lambda_{n} \sim a_{n+2}^{4} n^{-2}$. In other words, $\lambda_{n} \sim$ constant. Hence, it can be said that the Hermite-Sobolev coherent inner products are self-balanced.

\section{Freud-Sobolev orthogonal polynomials}

We are going to test the arguments developed in the previous section for the case of a Sobolev inner product related to Freud weights. The simplest example corresponds to $W_{0}^{2}(x)=W_{1}^{2}(x)=e^{-x^{2}}$, but this is a trivial case since for any choice of 
$\lambda_{n}$, the Sobolev orthogonal polynomial $S_{n, \lambda_{n}}$ is the $n$th monic Hermite polynomial. In this section, we show asymptotics for the Sobolev orthogonal polynomials with $W^{2}(x)=W_{0}^{2}(x)=W_{1}^{2}(x)=\exp \left(-x^{4}\right)$.

Throughout the section, $\left(P_{n}\right)_{n \geq 0}$ denotes the sequence of monic polynomials orthogonal with respect to the weight $W^{2},\|\cdot\|$ stands for the $L^{2}\left(W^{2}\right)$-norm, and $S_{n, \lambda_{n}}$ is the monic polynomial which minimizes

$$
\left\langle Q_{n}, Q_{n}\right\rangle_{\lambda_{n}}=\int_{\mathbb{R}} Q_{n}^{2}(x) W^{2}(x) d x+\lambda_{n} \int_{\mathbb{R}}\left(Q_{n}^{\prime}\right)^{2}(x) W^{2}(x) d x
$$

in the class of all monic polynomials of degree $n$.

The Mhaskar-Rakhmanov-Saff numbers for $W(x)=\exp \left(-x^{4} / 2\right)$ satisfy $a_{n} \sim$ $n^{1 / 4}$, and therefore condition (6) holds for $W$. As we have explained in Section 2, to balance this Sobolev inner product we must take $\lambda_{n} n^{2} \sim a_{n+1}^{2}$, that is, $\lambda_{n}$ like $n^{-3 / 2}$ when $n \rightarrow \infty$.

Next, we study the asymptotic behavior of the ratio $\frac{S_{n, \lambda_{n}}}{P_{n}}$ showing that the choice of $\lambda_{n}$ provides the reasonable one in a sense we will explain later. For technical reasons some additional constraints should be imposed on the parameters $\lambda_{n}$, so we deal with a decreasing sequence $\left(\lambda_{n}\right)$ of positive real numbers such that

$$
\lim _{n \rightarrow \infty} n^{3 / 2} \lambda_{n}=L \in[0,+\infty]
$$

and

$$
\lim _{n \rightarrow \infty} n^{7 / 4}\left(\lambda_{n-2}-\lambda_{n}\right)=0=\lim _{n \rightarrow \infty} n^{1 / 4}\left(\frac{\lambda_{n-2}}{\lambda_{n}}-1\right) .
$$

Notice that the sequence $\lambda_{n}=n^{-3 / 2}$ satisfies (7) and (8).

Proposition 1. Let $\left(\lambda_{n}\right)$ be a decreasing sequence of positive real numbers which satisfies $\frac{\lambda_{n-2}}{\lambda_{n}} \rightarrow 1$ and $n^{3 / 2} \lambda_{n} \rightarrow L \in[0,+\infty]$. Then

$$
\kappa(L):=\lim _{n \rightarrow \infty} \frac{\left\langle S_{n, \lambda_{n}}, S_{n, \lambda_{n}}\right\rangle_{\lambda_{n}}}{\left\|P_{n}\right\|^{2}}= \begin{cases}1 & \text { if } L=0, \\ \frac{2 L}{\sqrt{3}} \varphi\left(\frac{20 L+3 \sqrt{3}}{12 L}\right) & \text { if } 0<L<+\infty, \\ +\infty & \text { if } L=+\infty\end{cases}
$$

where $\varphi(x)=x+\sqrt{x^{2}-1}$.

Proof. We consider the Fourier expansion of the polynomial $P_{n}$ in terms of the basis $\left(S_{m, \lambda_{n}}\right)_{m \geq 0}$. Because the weight $e^{-x^{4}}$ is a symmetric function, we have

$$
P_{n}(z)=S_{n, \lambda_{n}}(z)+\sum_{j=0}^{n-2} \alpha_{j}\left(\lambda_{n}\right) S_{j, \lambda_{n}}(z)
$$

where

$$
\alpha_{j}\left(\lambda_{n}\right)=\frac{\left\langle P_{n}, S_{j, \lambda_{n}}\right\rangle_{\lambda_{n}}}{\left\langle S_{j, \lambda_{n}}, S_{j, \lambda_{n}}\right\rangle_{\lambda_{n}}}=\frac{\lambda_{n} \int_{\mathbb{R}} P_{n}^{\prime}(x) S_{j, \lambda_{n}}^{\prime}(x) e^{-x^{4}} d x}{\left\langle S_{j, \lambda_{n}}, S_{j, \lambda_{n}}\right\rangle_{\lambda_{n}}}, \quad 0 \leq j \leq n-2 .
$$

Since the orthogonal polynomials $P_{n}$ satisfy the following structure relation (see [12]),

$$
P_{n}^{\prime}(z)=n P_{n-1}(z)+\frac{4\left\|P_{n}\right\|^{2}}{\left\|P_{n-3}\right\|^{2}} P_{n-3}(z)
$$


the coefficients $\alpha_{j}\left(\lambda_{n}\right)$ vanish for $0 \leq j<n-2$. For $j=n-2$ we get

$$
\alpha_{n-2}\left(\lambda_{n}\right)=\frac{4(n-2) \lambda_{n}\left\|P_{n}\right\|^{2}}{\left\langle S_{n-2, \lambda_{n}}, S_{n-2, \lambda_{n}}\right\rangle_{\lambda_{n}}},
$$

and therefore

$$
P_{n}(z)=S_{n, \lambda_{n}}(z)+\alpha_{n-2}\left(\lambda_{n}\right) S_{n-2, \lambda_{n}}(z), \quad n \geq 3 .
$$

From now on, we will write $\kappa_{m}\left(\lambda_{n}\right)=\left\langle S_{m, \lambda_{n}}, S_{m, \lambda_{n}}\right\rangle_{\lambda_{n}}, n, m \geq 0$.

Now, observe that (12) leads to

$$
\begin{aligned}
& \kappa_{n}\left(\lambda_{n}\right)=\left\langle P_{n}-\alpha_{n-2}\left(\lambda_{n}\right) S_{n-2, \lambda_{n}}, P_{n}-\alpha_{n-2}\left(\lambda_{n}\right) S_{n-2, \lambda_{n}}\right\rangle_{\lambda_{n}} \\
& =\int_{\mathbb{R}}\left[\left(P_{n}-\alpha_{n-2}\left(\lambda_{n}\right) S_{n-2, \lambda_{n}}\right)^{2}+\lambda_{n}\left(P_{n}^{\prime}-\alpha_{n-2}\left(\lambda_{n}\right) S_{n-2, \lambda_{n}}^{\prime}\right)^{2}\right] e^{-x^{4}} d x .
\end{aligned}
$$

Then, using (10) and the orthogonality of $P_{n}$ with respect to the weight function $e^{-x^{4}}$, we have:

$$
\begin{aligned}
\kappa_{n}\left(\lambda_{n}\right)= & \left\|P_{n}\right\|^{2}+n^{2} \lambda_{n}\left\|P_{n-1}\right\|^{2}-8(n-2) \lambda_{n} \alpha_{n-2}\left(\lambda_{n}\right)\left\|P_{n}\right\|^{2} \\
& +16 \lambda_{n} \frac{\left\|P_{n}\right\|^{4}}{\left\|P_{n-3}\right\|^{2}}+\alpha_{n-2}^{2}\left(\lambda_{n}\right) \kappa_{n-2}\left(\lambda_{n}\right) .
\end{aligned}
$$

Taking into account the value of $\alpha_{n-2}\left(\lambda_{n}\right)$ given by (11), we get

$$
\kappa_{n}\left(\lambda_{n}\right)=\left\|P_{n}\right\|^{2}\left(B_{n}\left(\lambda_{n}\right)-A_{n}\left(\lambda_{n}\right) \frac{\left\|P_{n-2}\right\|^{2}}{\kappa_{n-2}\left(\lambda_{n-2}\right)}\right), n \geq 3,
$$

where

$$
\begin{aligned}
& A_{n}\left(\lambda_{n}\right)=16 \lambda_{n}^{2}(n-2)^{2} \frac{\kappa_{n-2}\left(\lambda_{n-2}\right)}{\kappa_{n-2}\left(\lambda_{n}\right)} \frac{\left\|P_{n}\right\|^{2}}{\left\|P_{n-2}\right\|^{2}} \\
& B_{n}\left(\lambda_{n}\right)=1+\lambda_{n} n^{2} \frac{\left\|P_{n-1}\right\|^{2}}{\left\|P_{n}\right\|^{2}}+16 \lambda_{n} \frac{\left\|P_{n}\right\|^{2}}{\left\|P_{n-3}\right\|^{2}}
\end{aligned}
$$

Next, we study $\lim _{n} B_{n}\left(\lambda_{n}\right)$ and $\lim _{n} A_{n}\left(\lambda_{n}\right)$. First, recall that the polynomials $P_{n}$ satisfy (see [12])

$$
\lim _{n \rightarrow \infty} \frac{\sqrt{n}\left\|P_{n-1}\right\|^{2}}{\left\|P_{n}\right\|^{2}}=2 \sqrt{3}
$$

On the other hand, $\lim _{n \rightarrow \infty} \frac{\kappa_{n-2}\left(\lambda_{n-2}\right)}{\kappa_{n-2}\left(\lambda_{n}\right)}=1$. Indeed, from the assumptions on $\lambda_{n}$ and using the extremal property of the norms of monic orthogonal polynomials, we have

$$
\begin{aligned}
\kappa_{n-2}\left(\lambda_{n}\right) & \leq \kappa_{n-2}\left(\lambda_{n-2}\right) \leq\left\langle S_{n-2, \lambda_{n}}, S_{n-2, \lambda_{n}}\right\rangle_{\lambda_{n-2}} \\
& =\frac{\lambda_{n-2}}{\lambda_{n}}\left[\frac{\lambda_{n}}{\lambda_{n-2}}\left\|S_{n-2, \lambda_{n}}\right\|^{2}+\lambda_{n}\left\|S_{n-2, \lambda_{n}}^{\prime}\right\|^{2}\right] \leq \frac{\lambda_{n-2}}{\lambda_{n}} \kappa_{n-2}\left(\lambda_{n}\right) .
\end{aligned}
$$

Since $\frac{\lambda_{n-2}}{\lambda_{n}} \rightarrow 1$, it follows that

$$
\lim _{n \rightarrow \infty} \frac{\kappa_{n-2}\left(\lambda_{n-2}\right)}{\kappa_{n-2}\left(\lambda_{n}\right)}=1
$$


Firstly, let us suppose that $0 \leq L<+\infty$. Then from (15) and (14) we deduce that

$$
\lim _{n \rightarrow \infty} B_{n}\left(\lambda_{n}\right)=1+\frac{20}{9} \sqrt{3} L \text { and } \quad \lim _{n \rightarrow \infty} A_{n}\left(\lambda_{n}\right)=\frac{4}{3} L^{2}
$$

To obtain (9), observe that, denoting $s_{n}=\kappa_{n}\left(\lambda_{n}\right) /\left\|P_{n}\right\|^{2}$, (13) becomes

$$
s_{n}=B_{n}\left(\lambda_{n}\right)-A_{n}\left(\lambda_{n}\right) \frac{1}{s_{n-2}} .
$$

Writing (17) for even indices and introducing a new sequence $\left(q_{n}\right)$ by means of $q_{n+1}=s_{2 n} q_{n}$, the above difference equation becomes

$$
q_{n+1}-B_{2 n}\left(\lambda_{2 n}\right) q_{n}+A_{2 n}\left(\lambda_{2 n}\right) q_{n-1}=0,
$$

whose characteristic equation

$$
q^{2}-\left(1+\frac{20}{9} \sqrt{3} L\right) q+\frac{4}{3} L^{2}=0
$$

has two simple and real roots with distinct moduli. Thus, Poincaré's Theorem (see, e.g., [10]) assures that $\frac{q_{n+1}}{q_{n}}=s_{2 n}$ converges to a root of (18). The extremal property of the norms yields

$$
\kappa_{n}\left(\lambda_{n}\right) \geq\left\|P_{n}\right\|^{2}+\lambda_{n} n^{2}\left\|P_{n-1}\right\|^{2},
$$

and therefore, using (14),

$$
l=\lim _{n \rightarrow \infty} s_{2 n} \geq 1+\lim _{n \rightarrow \infty} \lambda_{2 n}(2 n)^{2} \frac{\left\|P_{2 n-1}\right\|^{2}}{\left\|P_{2 n}\right\|^{2}}=1+2 \sqrt{3} L .
$$

So, it follows easily that $l=\frac{1}{18}\left[9+20 \sqrt{3} L+\sqrt{768 L^{2}+360 \sqrt{3} L+81}\right]$. Notice that, if $L \in(0,+\infty)$, then $l=\frac{2 L}{\sqrt{3}} \varphi\left(\frac{20 L+3 \sqrt{3}}{12 L}\right)$.

In a similar way, we also prove that $s_{2 n+1}$ converges to $l$. As a conclusion, there exists $\lim _{n} s_{n}=l=\kappa(L)$, and so for $L \in[0,+\infty)$, the proposition follows.

To finish the proof, let us now assume that $L=+\infty$. From (15) and (14) we have

$$
\lim _{n \rightarrow \infty} \frac{A_{n}\left(\lambda_{n}\right)}{\left(\lambda_{n} n^{3 / 2}\right)^{2}}=\frac{4}{3} \quad \text { and } \quad \lim _{n \rightarrow \infty} \frac{B_{n}\left(\lambda_{n}\right)}{\lambda_{n} n^{3 / 2}}=\frac{20}{9} \sqrt{3} .
$$

Upon applying the same technique as in the case $L<+\infty$ and replacing $s_{n}$ by $s_{n} /\left(\lambda_{n} n^{3 / 2}\right)$, we obtain

$$
\lim _{n \rightarrow \infty} \frac{s_{n}}{\lambda_{n} n^{3 / 2}}=\lim _{n \rightarrow \infty} \frac{\kappa_{n}\left(\lambda_{n}\right)}{\lambda_{n} n^{3 / 2}\left\|P_{n}\right\|^{2}}=2 \sqrt{3} .
$$

Clearly, $\frac{\kappa_{n}\left(\lambda_{n}\right)}{\left\|P_{n}\right\|^{2}} \rightarrow+\infty$ when $n$ tends to infinity and we conclude our statement.

The main result of this section is the following:

Theorem 1. Let $\left(\lambda_{n}\right)$ be a decreasing sequence of positive real numbers such that $\lim _{n} n^{7 / 4}\left(\lambda_{n-2}-\lambda_{n}\right)=0=\lim _{n} n^{1 / 4}\left(\frac{\lambda_{n-2}}{\lambda_{n}}-1\right)$. If

$$
\lim _{n \rightarrow \infty} n^{3 / 2} \lambda_{n}=L \in[0,+\infty]
$$


then

$$
\lim _{n \rightarrow \infty} \frac{S_{n, \lambda_{n}}(z)}{P_{n}(z)}= \begin{cases}1 & \text { if } L=0, \\ \frac{1}{1-\left[\varphi\left(\frac{20 L+3 \sqrt{3}}{12 L}\right)\right]^{-1}} & \text { if } 0<L<+\infty, \\ 3 / 2 & \text { if } L=+\infty\end{cases}
$$

holds uniformly on compact subsets of $\mathbb{C} \backslash \mathbb{R}$.

Remarks. 1. The choice $\lambda_{n} \equiv$ constant, which corresponds to a nonbalanced inner product, is a particular case of $L=+\infty$, and then Theorem 1 recovers the result already obtained in $[3]$.

2. When $L \in(0,+\infty)$ the above result also has the following reading. Write

$$
\langle P, Q\rangle_{\lambda_{n}}=\int_{\mathbb{R}} P(x) Q(x) W^{2}(x) d x+\lambda_{n} \int_{\mathbb{R}} P^{\prime}(x) Q^{\prime}(x)\left[L W^{2}(x)\right] d x .
$$

If $\lambda_{n}=n^{-3 / 2}(1+o(1))$, then $\lim _{n \rightarrow \infty} \frac{S_{n, \lambda_{n}}}{P_{n}}$ depends on $L$, that is, on the ratio of the weights.

However, for any other choice of $\lambda_{n}$ 's, the dependence on $L$ disappears, in particular for $\lambda_{n}=n^{-2}$ (the right choice in the bounded case) and for $\lambda_{n} \equiv$ constant (the nonbalanced case). This shows that our selection of $\lambda_{n}$ is accurate since the asymptotic behavior of Sobolev orthogonal polynomials $S_{n, \lambda_{n}}$ depends on both measures.

To prove Theorem 1 we will use the following result on the strong asymptotics of $P_{n}$ which appears in [6, Section 3]:

$$
\lim _{n \rightarrow \infty} \frac{P_{n}(z)}{\left\|P_{n}\right\|} \frac{D_{n}(z)}{\varphi^{n+1 / 2}\left(z / a_{n}\right)}=\frac{1}{\sqrt{2 \pi}}
$$

uniformly on compact subsets of $\mathbb{C} \backslash \mathbb{R}$. Here, $a_{n}$ are the Mhaskar-RakhmanovSaff numbers associated with the weight function $W, \varphi(z)=z+\sqrt{z^{2}-1}$ is the conformal mapping from $\mathbb{C} \backslash[-1,1]$ onto the exterior of the unit circle, and

$$
D_{n}(z)=\exp \left(\frac{\sqrt{z^{2}-a_{n}^{2}}}{2 \pi} \int_{-a_{n}}^{a_{n}} \frac{-t^{4}}{(z-t) \sqrt{a_{n}^{2}-t^{2}}} d t\right), \quad z \in \mathbb{C} \backslash\left[-a_{n}, a_{n}\right] .
$$

We would like to remark that, for $z \in \mathbb{C} \backslash\left[-a_{n}, a_{n}\right]$,

$$
D_{n}(z)=D\left(\frac{1}{\varphi\left(z / a_{n}\right)}, W_{n}^{2}\right),
$$

where $W_{n}^{2}$ is the weight function on the unit circle $\mathbb{T}$ defined by

$$
W_{n}^{2}\left(e^{i \theta}\right)=W^{2}\left(a_{n} \cos \theta\right), \quad \theta \in[-\pi, \pi]
$$

and

$$
D\left(w, W_{n}^{2}\right)=\exp \left(\frac{1}{2 \pi} \int_{0}^{2 \pi} \frac{e^{i \theta}+w}{e^{i \theta}-w} \log W_{n}(\theta) d \theta\right), \quad|w|<1 .
$$

It is well known that $D\left(., W_{n}^{2}\right)$ is holomorphic in the open unit disk $\mathbb{D}$, belongs to the Hardy space $H^{2}(\mathbb{D})$, and satisfies:

(1) $D\left(w, W_{n}^{2}\right) \neq 0, \quad$ for $w \in \mathbb{D}$,

(2) $D\left(0, W_{n}^{2}\right)>0$, 
(3) for almost every $\zeta$ in the unit circle, $D\left(., W_{n}^{2}\right)$ has nontangential boundary values $D\left(\zeta, W_{n}^{2}\right)$ such that $\left|D\left(\zeta, W_{n}^{2}\right)\right|^{2}=W_{n}^{2}(\zeta)$

(see, for instance, 13]). 11.

Next, we prove a technical result that will also be used in the proof of Theorem

Lemma 1. Assume that the sequence $\left(\lambda_{n}\right)$ satisfies the same conditions as in Theorem 1]. Then

$$
\lim _{n \rightarrow \infty} \frac{S_{n, \lambda_{n-2}}(z)-S_{n, \lambda_{n}}(z)}{P_{n}(z)}=0,
$$

uniformly on compact subsets of $\mathbb{C} \backslash \mathbb{R}$.

Proof. On account of (20), it suffices to prove that

$$
\lim _{n \rightarrow \infty} \frac{S_{n, \lambda_{n-2}}(z)-S_{n, \lambda_{n}}(z)}{\left\|P_{n}\right\| \varphi^{n+1 / 2}\left(z / a_{n}\right)} D_{n}(z)=0
$$

uniformly on compact subsets of $\mathbb{C} \backslash \mathbb{R}$. To see this we will prove:

i) for every compact set $K$ in $\mathbb{C} \backslash \mathbb{R}$, there exists a constant $M_{K}$, not depending on $n$, such that for $n$ large enough,

$$
\begin{aligned}
& \sup _{z \in K}\left|\frac{S_{n, \lambda_{n-2}}(z)-S_{n, \lambda_{n}}(z)}{\left\|P_{n}\right\| \varphi^{n+1 / 2}\left(z / a_{n}\right)} D_{n}(z)\right|^{2} \\
& \leq M_{K} a_{n} \int_{-a_{n}}^{a_{n}} \frac{\left|S_{n, \lambda_{n-2}}(x)-S_{n, \lambda_{n}}(x)\right|^{2}}{\left\|P_{n}\right\|^{2}} W^{2}(x) d x
\end{aligned}
$$

and

ii)

$$
\lim _{n \rightarrow \infty} a_{n} \int_{-a_{n}}^{a_{n}} \frac{\left|S_{n, \lambda_{n-2}}(x)-S_{n, \lambda_{n}}(x)\right|^{2}}{\left\|P_{n}\right\|^{2}} W^{2}(x) d x=0 .
$$

The key idea for proving $i)$ is to use the conformal mapping $\varphi\left(z / a_{n}\right)$ which applies $\mathbb{C} \backslash\left[-a_{n}, a_{n}\right]$ onto $\Omega=\{z \in \mathbb{C} ;|z|>1\}$, and the Cauchy integral representation for functions in $H^{2}(\Omega)$. Here, $H^{2}(\Omega)$ denotes the space of analytic functions $f$ in $\Omega$, with limit at $\infty$ and such that $f\left(\frac{1}{z}\right)$ belongs to the Hardy space $H^{2}(\mathbb{D})$. From the Cauchy integral representation for functions in $H^{2}(\mathbb{D})$, see [13], we have that if $f \in H^{2}(\Omega)$, then

$$
f(w)=-\frac{1}{2 \pi i} \int_{|\zeta|=1} \frac{f^{*}(\zeta)}{\zeta-w} \frac{w}{\zeta} d \zeta, \quad w \in \Omega
$$

where $f^{*}(\zeta)=\lim _{r \searrow 1} f(r \zeta)$ and the unit circle is positively oriented.

In order to prove $i$ ), given a compact set $K$ in $\mathbb{C} \backslash \mathbb{R}$, there exists an absolute constant $C_{K}>0$ such that

$$
\left|\sqrt{z^{2}-a_{n}^{2}}\right| \geq C_{K}, \quad \forall z \in K, \quad \forall n \geq 0
$$


Therefore, if $z \in K$,

$$
\begin{aligned}
& \left|\frac{S_{n, \lambda_{n-2}}(z)-S_{n, \lambda_{n}}(z)}{\left\|P_{n}\right\| \varphi^{n+1 / 2}\left(z / a_{n}\right)} D\left(\frac{1}{\varphi\left(z / a_{n}\right)}, W_{n}^{2}\right)\right|^{2} \\
& \leq \frac{1}{C_{K}}\left|\frac{S_{n, \lambda_{n-2}}(z)-S_{n, \lambda_{n}}(z)}{\left\|P_{n}\right\| \varphi^{n+1 / 2}\left(z / a_{n}\right)} D\left(\frac{1}{\varphi\left(z / a_{n}\right)}, W_{n}^{2}\right)\right|^{2}\left|\sqrt{z^{2}-a_{n}^{2}}\right| \\
& =\frac{1}{C_{K}}\left|F_{n}(w)\right|,
\end{aligned}
$$

where

$$
F_{n}(w)=\left[\frac{\left(S_{n, \lambda_{n-2}}-S_{n, \lambda_{n}}\right)\left(a_{n} \varphi^{-1}(w)\right)}{\left\|P_{n}\right\| w^{n+1 / 2}} D\left(\frac{1}{w}, W_{n}^{2}\right)\right]^{2} a_{n} \sqrt{\left(\varphi^{-1}(w)\right)^{2}-1},
$$

with $w=\varphi\left(z / a_{n}\right)$.

It is easy to check that $F_{n} \in H^{2}(\Omega)$ and its boundary values are

$$
F_{n}^{*}\left(e^{i \theta}\right)=\frac{\left(S_{n, \lambda_{n-2}}-S_{n, \lambda_{n}}\right)^{2}\left(a_{n} \cos \theta\right)}{\left\|P_{n}\right\|^{2} e^{i(2 n+1) \theta}} W^{2}\left(a_{n} \cos \theta\right) a_{n} \sqrt{\cos ^{2} \theta-1} .
$$

Moreover, if we denote by $K_{n}=\left\{\varphi\left(z / a_{n}\right) ; z \in K\right\}$, straightforward computations yield that there exists an absolute constant $A_{K}>0$ such that the distance between $K_{n}$ and the unit circle satisfies $d\left(K_{n}, \mathbb{T}\right) \geq A_{K} / a_{n}$ for $n$ large enough. Then, from the integral formula (22) applied to $F_{n}$ we have for $w \in K_{n}$,

$$
\begin{aligned}
\left|F_{n}(w)\right| & \leq B_{K} a_{n} \int_{|\zeta|=1}\left|F_{n}^{*}(\zeta)\right||d \zeta| \\
& =B_{K} a_{n} \int_{-\pi}^{\pi} \frac{\left(S_{n, \lambda_{n-2}}-S_{n, \lambda_{n}}\right)^{2}\left(a_{n} \cos \theta\right)}{\left\|P_{n}\right\|^{2}} W^{2}\left(a_{n} \cos \theta\right) a_{n}|\sin \theta| d \theta \\
& =2 B_{K} a_{n} \int_{-a_{n}}^{a_{n}} \frac{\left(S_{n, \lambda_{n-2}}-S_{n, \lambda_{n}}\right)^{2}(x)}{\left\|P_{n}\right\|^{2}} W^{2}(x) d x
\end{aligned}
$$

where $B_{K}$ is an absolute positive constant depending only on $K$. So $i$ ) is proved.

In order to deduce $i i)$, observe that

$$
\begin{aligned}
& \int_{\mathbb{R}}\left|S_{n, \lambda_{n-2}}(x)-S_{n, \lambda_{n}}(x)\right|^{2} W^{2}(x) d x \leq\left\langle S_{n, \lambda_{n-2}}-S_{n, \lambda_{n}}, S_{n, \lambda_{n-2}}-S_{n, \lambda_{n}}\right\rangle_{\lambda_{n}} \\
& =\left\langle S_{n, \lambda_{n-2}}, S_{n, \lambda_{n-2}}\right\rangle_{\lambda_{n}}-\left\langle S_{n, \lambda_{n}}, S_{n, \lambda_{n}}\right\rangle_{\lambda_{n}} \\
& =\kappa_{n}\left(\lambda_{n-2}\right)+\left(\lambda_{n}-\lambda_{n-2}\right) \int_{\mathbb{R}}\left|S_{n, \lambda_{n-2}}^{\prime}(x)\right|^{2} W^{2}(x) d x-\kappa_{n}\left(\lambda_{n}\right) \\
& \leq \kappa_{n}\left(\lambda_{n-2}\right)-\kappa_{n}\left(\lambda_{n}\right) .
\end{aligned}
$$

Therefore, for every $n$ we get

$$
a_{n} \int_{-a_{n}}^{a_{n}} \frac{\left|S_{n, \lambda_{n-2}}(x)-S_{n, \lambda_{n}}(x)\right|^{2}}{\left\|P_{n}\right\|^{2}} W^{2}(x) d x \leq a_{n} \frac{\kappa_{n}\left(\lambda_{n-2}\right)-\kappa_{n}\left(\lambda_{n}\right)}{\left\|P_{n}\right\|^{2}} .
$$

Finally, since $a_{n} \sim n^{1 / 4}$ it is enough to prove that $n^{1 / 4} \frac{\kappa_{n}\left(\lambda_{n-2}\right)-\kappa_{n}\left(\lambda_{n}\right)}{\left\|P_{n}\right\|^{2}}$ tends to 0 when $n$ tends to infinity. Indeed, since

$$
0 \leq \kappa_{n}\left(\lambda_{n-2}\right)-\kappa_{n}\left(\lambda_{n}\right) \leq\left(1-\frac{\lambda_{n}}{\lambda_{n-2}}\right) \kappa_{n}\left(\lambda_{n-2}\right),
$$


we have

$$
n^{1 / 4} \frac{\kappa_{n}\left(\lambda_{n-2}\right)-\kappa_{n}\left(\lambda_{n}\right)}{\left\|P_{n}\right\|^{2}} \leq n^{1 / 4}\left(1-\frac{\lambda_{n}}{\lambda_{n-2}}\right) \frac{\kappa_{n}\left(\lambda_{n}\right)}{\left\|P_{n}\right\|^{2}} \frac{\kappa_{n}\left(\lambda_{n-2}\right)}{\kappa_{n}\left(\lambda_{n}\right)} .
$$

Now, taking into account that $\lim _{n} \frac{\kappa_{n}\left(\lambda_{n-2}\right)}{\kappa_{n}\left(\lambda_{n}\right)}=1$, it suffices to keep in mind Proposition 1. (8), and (19) to conclude $i i$ ) and therefore the proof of the lemma.

Proof of Theorem 1. The algebraic relation between the polynomials $P_{n}$ and the Sobolev polynomials given by (12) can be rewritten for $\lambda_{n-2}$ as

$$
\begin{aligned}
P_{n}(z) & =S_{n, \lambda_{n-2}}(z)+\alpha_{n-2}\left(\lambda_{n-2}\right) S_{n-2, \lambda_{n-2}}(z) \\
& =S_{n, \lambda_{n}}(z)+S_{n, \lambda_{n-2}}(z)-S_{n, \lambda_{n}}(z)+\alpha_{n-2}\left(\lambda_{n-2}\right) S_{n-2, \lambda_{n-2}}(z) .
\end{aligned}
$$

Then, dividing both sides of the above expression by $P_{n}(z)$, we obtain

$$
f_{n}(z)=b_{n}(z) f_{n-2}(z)+c_{n}(z), \quad z \in \mathbb{C} \backslash \mathbb{R},
$$

where

$$
\begin{aligned}
& f_{n}(z)=\frac{S_{n, \lambda_{n}}(z)}{P_{n}(z)}, \quad b_{n}(z)=-\alpha_{n-2}\left(\lambda_{n-2}\right) \frac{P_{n-2}(z)}{P_{n}(z)}, \\
& c_{n}(z)=1-\frac{S_{n, \lambda_{n-2}}(z)-S_{n, \lambda_{n}}(z)}{P_{n}(z)} .
\end{aligned}
$$

Firstly, we study the limits of the sequences $\left(b_{n}(z)\right)$ and $\left(c_{n}(z)\right)$. As a consequence of Lemma 1 we know that

$$
\lim _{n \rightarrow \infty} c_{n}(z)=1
$$

uniformly on compact subsets of $\mathbb{C} \backslash \mathbb{R}$.

With regard to $\left(b_{n}(z)\right)$, if $L \in[0,+\infty)$, from Proposition 1 and (14),

$$
\frac{\alpha_{n-2}\left(\lambda_{n-2}\right)}{\sqrt{n-2}}=4 \lambda_{n-2}(n-2)^{3 / 2} \frac{\left\|P_{n-2}\right\|^{2}}{\kappa_{n-2}\left(\lambda_{n-2}\right)} \frac{\left\|P_{n}\right\|^{2}}{(n-2)\left\|P_{n-2}\right\|^{2}} \rightarrow \frac{L}{3 \kappa(L)} .
$$

Moreover, for the monic polynomials $P_{n}$ it is known (see [6]) that

$$
\lim _{n \rightarrow \infty} \frac{\sqrt{n-2} P_{n-2}(z)}{P_{n}(z)}=-2 \sqrt{3},
$$

uniformly on compact subsets of $\mathbb{C} \backslash \mathbb{R}$. Both results lead to

$$
\lim _{n \rightarrow \infty} b_{n}(z)=\frac{2 L}{\sqrt{3} \kappa(L)}= \begin{cases}0 & \text { if } L=0 \\ \frac{1}{\varphi\left(\frac{20 L+3 \sqrt{3}}{12 L}\right)} & \text { if } 0<L<+\infty\end{cases}
$$

uniformly on compact subsets of $\mathbb{C} \backslash \mathbb{R}$. In the case $L=+\infty$, using formula (19) we get $\lim _{n} b_{n}(z)=1 / 3$, uniformly on compact subsets of $\mathbb{C} \backslash \mathbb{R}$.

Finally, observe that the functions $f_{n}, b_{n}$ and $c_{n}$ are analytic in $\mathbb{C} \backslash \mathbb{R}$. Since for $L \in[0,+\infty]$ we have $\lim _{n} b_{n}(z)=b_{L}$, with $\left|b_{L}\right|<1$, and $\lim _{n} c_{n}(z)=1$ uniformly on compact subsets of $\mathbb{C} \backslash \mathbb{R}$, we can deduce that

$$
\lim _{n \rightarrow \infty} f_{n}(z)=\frac{1}{1-b_{L}}
$$

uniformly on compact subsets of $\mathbb{C} \backslash \mathbb{R}$. Indeed, for a fixed compact set $K \subset \mathbb{C} \backslash \mathbb{R}$, there exist constants $r \in(0,1), R>1$ and a positive integer number $n_{0}$ such that

$$
\left|b_{n}(z)\right| \leq r, \quad\left|c_{n}(z)\right| \leq R, \quad \text { for } \quad n \geq n_{0}, \quad z \in K
$$


Thus

$$
\left|f_{n}(z)\right| \leq r\left|f_{n-2}(z)\right|+R, \quad \text { for } \quad n \geq n_{0}, \quad z \in K,
$$

and therefore we deduce that the sequence $\left(f_{n}\right)$ is uniformly bounded on compact subsets of $\mathbb{C} \backslash \mathbb{R}$.

From (23), we can write

$$
f_{n}(z)-\frac{1}{1-b_{L}}=b_{L}\left[f_{n-2}(z)-\frac{1}{1-b_{L}}\right]+\varepsilon_{n}(z)
$$

with

$$
\varepsilon_{n}(z)=\left(b_{n}(z)-b_{L}\right) f_{n-2}(z)+c_{n}(z)-1 .
$$

Notice that $\lim _{n} \varepsilon_{n}(z)=0$, uniformly on compact subsets of $\mathbb{C} \backslash \mathbb{R}$. From the fact $\left|b_{L}\right|<1$, it is easy to deduce that

$$
\lim _{n \rightarrow \infty} f_{n}(z)=\frac{1}{1-b_{L}}
$$

uniformly on compact subsets of $\mathbb{C} \backslash \mathbb{R}$. Taking into account the value of $b_{L}$ with $L \in[0,+\infty]$, the theorem is proved.

\section{ACKNOWLEDGEMENTS}

We express our gratitude to Professor Andrei Martínez-Finkelshtein for his suggestions and fine comments on this research. We also thank the referee for his observations and comments which have improved this article.

\section{REFERENCES}

[1] M. Alfaro, A. Martínez-Finkelshtein and M. L. Rezola, Asymptotic Properties of Balanced Extremal Sobolev Polynomials: Coherent Case, J. Approx. Theory 100 (1999), 44-59. MR.1710552 (2001a:42022)

[2] M. Alfaro, J. J. Moreno-Balcázar, T. E. Pérez, M. A. Piñar, and M. L. Rezola, Asymptotics of Sobolev orthogonal polynomials for Hermite coherent pairs, J. Comput. Appl. Math. 133 (2001), 141-150. MR.1858274 (2002k:33005)

[3] A. Cachafeiro, F. Marcellán, and J. J. Moreno-Balcázar, On asymptotic properties of Freud-Sobolev orthogonal polynomials, J. Approx. Theory 125 (2003), 26-41. MR2016838 (2005e:33004)

[4] J. S. Geronimo, D. S. Lubinsky, and F. Marcellán, Asymptotics for Sobolev Orthogonal Polynomials for Exponential Weights, Constr. Approx. 22 (2005), 309-346. MR2164139 (2006c:41040)

[5] E. Levin and D. S. Lubinsky, Orthogonal Polynomials for Exponential Weights, SpringerVerlag, Berlin, 2001. MR1840714 (2002k:41001)

[6] G. López and E. A. Rakhmanov, Rational approximations, orthogonal polynomials and equilibrium distributions, (in) Lecture Notes in Mathematics, Vol. 1329, Springer-Verlag, Berlin, 1988, pp. 125-157. MR973424 (90d:41028)

[7] F. Marcellán and J. J. Moreno-Balcázar, Asymptotics and zeros of Sobolev orthogonal polynomials on unbounded supports, Acta Appl. Math. 94 (2006), 163-192. MR2273888 (2007i:42002)

[8] A. Martínez-Finkelshtein, Bernstein-Szegő's theorem for Sobolev orthogonal polynomials, Constr. Approx. 16 (2000), 73-84. MR 1848842(2002h:42053)

[9] A. Martínez-Finkelshtein, J. J. Moreno-Balcázar, T. E. Pérez, and M. A. Piñar, Asymptotics of Sobolev orthogonal polynomials for coherent pairs of measures, J. Approx. Theory 92 (1998), 280-293. MR.1604939 (98m:42038)

[10] L. M. Milne-Thomson, The Calculus of Finite Differences, MacMillan, New York, 1965. MR0043339 (13:245c)

[11] J. J. Moreno-Balcázar, Smallest zeros of some types of orthogonal polynomials: Asymptotics, J. Comput. Appl. Math. 179 (2005), 289-301. MR2134372 (2005m:42041) 
[12] P. Nevai, Orthogonal polynomials associated with exp $\left(-x^{4}\right)$. Volume 3 of Canad. Math. Soc. Conf. Proc., 263-285, 1983. MR729336 (85b:42012)

[13] W. Rudin, Real and Complex Analysis, McGraw-Hill, New York, 1986.

[14] E. B. Saff and V. Totik, Logarithmic Potentials with External Fields. Volume 316 of Grundlehren der Mathematischen Wissenschaften. Springer-Verlag, Berlin, 1997. MR 1485778 (99h:31001)

[15] H. Stahl and V. Totik, General Orthogonal Polynomials. Volume 43 of Encyclopedia of Mathematics and its Applications. Cambridge University Press, Cambridge, 1992. MR.1163828 (93d:42029)

Departamento de Matemáticas and iUma, Universidad de Zaragoza, c/Pedro CerBuna 12, 50009 Zaragoza, Spain

Departamento de Estadística y Matemática Aplicada, Universidad de Almería, La Canada de San Urbano, 04120 Almeria, Spain - And - Instituto Carlos I de Física Teórica y Computacional, Universidad de Granada, Granada, Spain

Departamento de Matemáticas and iUma, Universidad de Zaragoza, c/Pedro CerBUNA 12, 50009 Zaragoza, SPAIN

Departamento de Matemáticas and iUma, Universidad de Zaragoza, c/Pedro CerBuna 12, 50009 Zaragoza, Spain

E-mail address: rezola@unizar.es 